Introduction Acute renal failure is a severe complication in neonatal intensive care medicine. The only therapeutic option for renal replacement therapy in small infants or premature babies with acute renal failure is peritoneal dialysis. In this paper two case reports of sealing an untight peritoneal catheter with cyanoacrylate glue will be presented.

Patient 1: Premature infant of $34+2$ weeks gestational age. Dorsal cloaca malformation with megacystis and megaureter, peritoneal dialysis for 13 days.

Patient 2: Term neonate of $40+4$ weeks gestational age with meconium plug syndrome and volvulus, double ileostomy, necrotising enterocolitis and Morbus Hirschsprung. Alagille syndrome with renal malformation, peritoneal dialysis for 46 days.

All catheters were extremely untight from the very beginning; effective dialysis was not possible. Sealing with fibrine glue was not efficient. Only sealing of the skin with cyanoacrylate glue allowed for effective peritoneal dialysis. Apart from gradually increasing irritation of the skin no serious side effects occurred. No dialysate entered the surrounding soft tissue although the subcutaneous part of the skin channel was always filled with dialysate.

Conclusion It is possible to seal a catheter for peritoneal dialysis quickly and effectively with cyanoacrylate glue without serious complications. The existence of an ileostomy did not interfere with the dialysis.

\section{P0-0665 THE RELATIONSIPS BETWEEN RISK FACTORS FOR HEARING IMPAIRMENT AND THE RESULTS OF NEWBORN HEARING SCREENING}

H Sarbay, S Guven, A Yazar, S Bozdag, S Akova, I Islek. Pedatrics, Umraniye Training and Research Hospital, Istanbul, Turkey

\subsection{6/archdischild-2014-307384.1306}

Background and aims Normal hearing in early infancy is essential forspeech, language, and social and emotional development of human beings. Congenital hearing loss has an impact on normal speech and language. Newborn hearing screenings has been proposed for the early diagnosis and treatment of infants with hearing loss, and thereby improve language outcomes in these babies. Our aim was to evaluate the relationships between risk factors for hearing impairment and auto acoustic emission (OAE) and auditory brainstem responses (ABR) results in patient's follow-up at Neonatal Clinic.

Material and methods OAE and ABR measurements were performed to identify infants with hearing loss. Thosenewborns who did not pass OAE test or passed the OAE test but had risk factorsfor hearing impairment were screened by an ABR. The risk factors for hearingloss have been evaluated according to the Joint Committee on Infant Hearing (JCIH), 2007.

Results 17 of 100 neonates in the group with risk factors failed OAE tests, of which 5 were also failed ABR test. 3 of 100 neonates in the group without risk factors failed OAE tests, of which 1 was also failed ABR test. When the test fails compared with the number of risk factors; $1 \%$ in neonates with no risk factors, $3.2 \%$ in neonates with 1 risk factor, $25 \%$ in the group with two risk factors, $100 \%$.of neonates with 3 risk factors were failed the screening.

Conclusions The increase in the number of risk factors significantly increases the failure rate of the ABR test.

\section{P0-0666 ACUPRESSURE FOR PRETERM INFANTS IN PAIN RELIEF}

${ }^{1}$ A Abbasoglu, ${ }^{2} \mathrm{~T}$ Cabioglu, ${ }^{3} \mathrm{E}$ Yapakci, ${ }^{1} \mathrm{~A}$ Ecevit, ${ }^{1} \mathrm{~A}$ Ecevit, ${ }^{1} \mathrm{AU}$ Tugcu, ${ }^{1} \mathrm{~A}$ Tarcan. ${ }^{1}$ Neonatology, Baskent University, Ankara, Turkey; ${ }^{2}$ Physiology, Baskent University, Ankara, Turkey; ${ }^{3}$ Neonatology, Ankara Güven Hospital, Ankara, Turkey

\subsection{6/archdischild-2014-307384.1307}

Background and aims Recent studies have shown that newborns remember and perceive the pain, and they have been shown to feel pain from intrauterine life. To avoid adverse effects of pharmacologic analgesic agents, non pharmacologic strategies to minimise neonatal procedural pain have been proposed. Acupressure is a complementary treatment that uses fingers and applies pressure to stimulate acupoints of the human body. We studied the analgesic effect of acupressure in preterm infants during heel prick blood draw.

Methods This study was carried out in a tertiary care neonatal unit at the Baskent University in Turkey. 32 infants born before the age of 37 weeks, and who did not have sepsis, any metabolic or genetic disease, and did not receive any medication for sedation or analgesia were included. The experimental group was given both routine care and acupressure. The control group only underwent routine care. Kunlun point (EX-HN3) and Taixi point (K3) was kneaded for 3 min before the procedure. All babies were scored according to the Premature Infant Pain Profile (PIPP) by a second researcher.

Results In both groups of infants enrolled in the study; gestational age, birth weight, postnatal day, the actual weights were similar $(\mathrm{p}>0.05)$. The procedure time and crying time in the acupressure group was significantly lower than other group ( $\mathrm{p}=$ 0.00). PIPP scores were not found different $(\mathrm{p}=0,046)$.

Conclusions In this study, applying acupressure did not change the PIPP score in preterm infants. More research should be done in different acupressure points for analgesic effect for preterm infants.

\section{PO-0667 RECOGNITION OF BILE COLOUR IN NEONATAL VOMIT}

H Abdelrhim. Neonates, North Bristol NHS Trust, Bristol, UK

\subsection{6/archdischild-2014-307384.1308}

Introduction Neonatal bilious vomiting is a surgical emergency until proved otherwise, and requires prompt investigation. Health workers who are unfamiliar with the colour of bile may over or under-diagnose the presence of bile in vomit or gastric aspirates.

Objective The aim of this study was to assess whether the nursing staff, midwives and doctors at a district general hospital, where the exposure to newborns with bilious vomiting is less common than in tertiary hospitals, are able to identify the colour of bile accurately.

Design A total of 163 participants of both parents and health workers were asked to identify bile colour from a colour-chart of 8 green/yellow colour options.

Setting Paediatric Department in Yeovil District Hospital from the first of April, 2013 to August, 2013.

The results Chi-Square goodness-of-fit tests and KolmogorovSmirnov two-sample tests were conducted to compare different groups and answers.

Showed that the colour of bile was correctly identified by $95.5 \%$ of medical staff, $91 \%$ of nurses, midwives $72 \%$ and only $16.5 \%$ of parents. Whereas $16 \%$ of doctors, $30.5 \%$ of nurses, midwives $28 \%$ and $34 \%$ of parents wrongly identified the 